Gynäk. Rdsch. 1976;16:I-V

\title{
Contents, Vol. 16, 1976
}

\section{Inhaltsverzeichnis}

Nr. 1

Kurzfassungen von Zeitschriftenartikeln

Dinermann, B.: Regionalisierung der Geburtshilfedienste

Ryan, G. jr.: Regionale Perinatalgesundheitsdienste. Beitrag zur Verbesserung der

Schwangerschaftsergebnisse 12

Fenig, J.; Arlen, M.; Livingston, S.F. und Levowitz, B.: Brustkrebs: Mikrokarzinom in der zweiten Brust? 28

Brinkley, D. und Haybittle, J.L.: Die Heilbarkeit von Brustkrebs

Originalarbeiten

Gitsch, E. und Kubista, E.: Ergebnisse der operativen Therapie des Ovarialkarzinoms der Jahre 1948-1968 35

Kunz, J.: Amenorrhö nach hormonaler Antikonzeption. Prophylaxe, Befunde und Therapie 47

Nr. 2

Kurzfassungen von Zeitschriftenartikeln

Chow, A.W.; Malkasian, K.L.; Marshall, J.B. und Guze, L.B.: Die Bakteriologie der akuten Entzündungen des Beckenraumes

Sweet, R.L.: Anaerobier-Infektionen des weiblichen Genitaltraktes

Favre, R.; Charpin-Taranger, C; Reboul, G.; Cabassu, E. und Carcassone, Y.: HodgkinKrankheit und Schwangerschaft 76

David, A.; Bar-David, E. und Serr, M.: Angeborene Scheidenaplasie. Klinische und psychologische Aspekte 83

Duenhoelter, J.A. und Gant, N.F.: Komplikationen nach Abortus-Einleitung mit Prostaglandin im 2. Trimenon 89

Inhaltsverzeichnis IV

Keller, B.; Wolff, F.; Leissner, P.; Swarcberg, R. und Foegle, J.: Einrisse im hinteren Blatt des Ligamentum latum. Laparoskopische Diagnose. Therapiemethoden und ihre Ërfolge bei 108 Fallen 94

Lentle, B.C.; Burns, P.; Dierich, H. und Jackson, F.I.: Knochenszintigraphie bei Brustkrebs 99

Origin alarbeiten

Kaufmann, E.: L'ablactation par anti-prolactine de synthèse 107 Janousek, F.: Fehlerquellen der histologischen und zytologischen Portiodiagnostik . 113 Baur, B. und Sidiropoulos, D.: Komplikationen mit Lokalanästhetika in der Geburtshilfe bzw. Perinatalperiode 122 Vilàghy, I.; Erb, H.; Bogdan, C; Wülausch, F. und Varga, L.: Ergebnisse der Inkontinenzoperationen von 1962 bis 1971 an der Frauenklinik Liestal 125 
Bogdan, C. und Varga, L.: Abklärung der Versager nach Inkontinenzoperationen ... 130 Maire, F. und Erb, H.: Postoperative Harnverhaltung und Spätresultate nach Inkonti nenzoperationen 135 Varga, L.; Bogdan, C. und Maire, F.: Vorgehen bei der Nachuntersuchung von Patientinnen mit Inkontinenzoperationen 138

Nr. 3

Kurzfassungen von Zeitschriftenartikeln Johansson, E.W.; Kaij, L.; Kullander, S.; Lenner, H.C.; Smnberg, L. und Astedt, B.: Einige Spätwirkungen nach beidseitiger Ovarektomie im Alter von 15-30 Jahren . 145 Saldana, L.R.; Schulman, H. und Lin, Chin-Chu: Routine-Amnioskopie am Tragzeitende 166 Wahl, P.; Quereux, C; Jakob, L.; Prudhomme, G. und Chaste, F.: Schockzustand der Mutter während einer Amnioskopie mit Auswirkung auf den Fetus 172

Pitkin, R.: Abdominale Uterusexstirpation bei übergewichtigen Frauen 175 Endler, G.C. und Moghissi, K.: Magenperf oration während der gynäkologischen Laparoskopie 181

Merger, M.R.: Schwangerschaftsabbruch. Bericht einer Kommission der Akademie der Medizin Paris 185

Block, M.F. und Rahhal, D.K.: Zervixinkompetenz. Ein diagnostisches und prognostisches Punktezahl-System 191

Übersichtsreferat

Vague, J.: Extragenitale Wirkungen der Sexualhormone bei der Frau 197

Originalarbeit

Holzer, E.; Haigermoser, A. und Picket, H.: Beidseitige metastatische Ovarialtumoren als akute Wochenbettkomplikation 211

Inhaltsverzeichnis $\mathrm{V}$

Nr. 4

Kurzfassungen von Zeitschriftenartikeln

Sorel, G.; Fafari, K.; Lash, A. und Stepto, R.: Akute Leukämie bei fortgeschrittenem Ovarialkarzinom nach Behandlung mit alkylierenden Substanzen 217

Juret, P.; Couette, E.; Mandard, A.M.; Carre, A.; Delozier, T.; Brune, D. und Vernhes, C: Alter bei der Menarche als Prognosefaktor bei Brustkrebs 223

Hallat, J.: Ektopische Schwangerschaft und Intrauterinspirale (siebzig Fälle) 229 Popper, N.S. und Moore, J.J.: E. co//-Sepsis bei einer Schwangeren mit Kupfer-7-IUD in utero 235

Originalarbeiten

Sidiropoulos, D.; Baur, B.; Gautschy, B.; Tonella, A. und Jann, F.X.: Klinische und Laborbefunde bei Frühgeborenen nach pränataler Betamethason-Verabreichung . 237 Muller, P.: Vorzeitige Ovarialerschöpfung 243

Baur, B.; Sidiropoulos, D.; Gigon, U.; Haldemann, R. und Ulrich, L.: Lokalanästhesie und Episiotomie 254

Eduah, SB. und Dreher, E.: Medikamentöse Beeinflussung des Urethralverschlusses bei apparativ gesicherter Stressinkontinenz der Frau 257

Sacha, E.; Gigon, U.; Dreher, E. und Jann, F.X.: Therapeutische intraamniale, transabdominale Abortinduktion im zweiten Trimenon mittels Prostaglandin F2 $\alpha$. . 261 
Thoma, R.; Ludin, H; Cassani, S. und Haag, B.: Die Bedeutung des lateralen Zystourethrogramms nach Green für die Indikationsstellung zur operativen Behandlung der Stressinkontinenz 264

Balint, Z.; Haefeli, H. und Haag, B.: Scheidenplastik mit Blasenhalsraffung nach KellyStoeckel mit gleichzeitiger vaginaler Hysterektomie zur Behandlung der Stress inkontinenz zweiten Grades 271

Aljinovic, A.; Gunti, J. und Pusterla, E.: Medikamentöse Ablaktation post partum. Vergleichsstudie zwischen Bromocriptin (Parlodel ${ }^{\circledR}$ ) und Methallenöstrol (Vallestril $\left.{ }^{\circledR}\right)$ sowie zwei Kombinationsvarianten der beiden Präparate 276

Varia 143

Autorenindex 281

Supplementum

Schweizerische Gesellschaft fur Gynäkologie

Bericht über die Jahresversammlung, 1.-3. Juli 1976 in Davos

Société Suisse de Gynécologie

Compte rendu de ГAssemblée annuelle, 1-3 juillet 1976 à Davos 